\title{
LXI. On an improvement in the analysis of equations
}

\section{J.R. Young}

To cite this article: J.R. Young (1849) LXI. On an improvement in the analysis of equations, Philosophical Magazine Series 3, 34:231, 413-415, DOI: 10.1080/14786444908646259

To link to this article: http://dx.doi.org/10.1080/14786444908646259

曲 Published online: 30 Apr 2009.

Submit your article to this journal $\pi$

Џll Article views: 2

Q View related articles $\sqsubset$ 


\section{[ 4113 ]}

LXI. On an Improvement in the Analysis of Equations. By J. R. Young, Professor of Mathematics in Belfast College*.

IN the analysis of numerical equations our chief difficulties 1 are with those of an even degree, into which equations of an odd degree may always be changed. The following brief sketch of an improved method of discussing such equations will, I think, be acceptable to the readers of the Philosophical Magazine, although I reserve minute details, as well as some important extensions, for a future communication.

Let

$$
x^{2 n}+a x^{2 n-1}+b x^{2 n-2}+c x^{2 n-3}+\ldots+k=0 .
$$

be any equation of an even degree with numerical coefficients; then the left-hand member may be decomposed into the following pair of conjugate factors, namely,

and

$$
x^{n}+\frac{1}{2} a x^{n-1}+\sqrt{ }\left\{\left(\frac{1}{4} a^{2}-b\right) x^{2 n-2}-c x^{2 n-3}-\ldots-k\right\},
$$

$$
x^{n}+\frac{1}{2} a x^{n-1}-\sqrt{ }\left\{\left(\frac{1}{4} a^{2}-b\right) x^{2 n-2}-c x^{2 n-3}-\ldots . . k\right\} ;
$$

and, consequently, if these be separately equated to zero, and either of the roots of the proposed equation substituted for $x$, one of these two new equations will always be satisfied. It follows, therefore, that when a real root is substituted, the expression under the radical, namely,

$$
\left(\frac{1}{4} a^{2}-b\right) x^{2 n-2}-c x^{2 n-3}-\ldots . .-k, \quad . \quad . \quad .
$$

must always be positive; otherwise a real expression, viz. that which precedes the radical, would neutralize an imaginary one.

Hence, if we perform the partial analysis of the equation (A) by the method of Budan, commonly called the method of Fourier, we may discuss the doubtful intervals, which that analysis leaves for further examination, by an appeal to the inferior polynomial (B). Applying the before-mentioned analysis to this, in reference to the doubtful intervals, we should infer that whenever, throughout any such interval, (B) was plus, the sought roots may be real; and that when, throughout, the sign was minus, they must be imaginary. When the sign of $(B)$ passed from plus to minus, or from minus to plus, in the interval, the doubt would remain; and

$$
\text { * Communicated by the Author. }
$$




\section{On an Improvement in the Analysis of Equations.}

our business would then be to take a portion of only the positive region of this interval, and to employ its limits in the corresponding interval of the original analysis: if two roots were still indicated, within these limits, we may suspect them to be real: if no roots be indicated, we must widen the positive interval in the second analysis: if the roots are real, we shall thus at length inclose them; but if no roots become indicated till we trench upon the negative portion of the interval in (B), we may be sure that the roots are imaginary.

At present I shall give but a single example in illustration. Let the equation be

$$
x^{4}-4 x^{3}-3 x+23=0,
$$

the complete analysis of which, by the method of Fourier, is attended with a good deal of trouble. (See Analyse des Equations, p. 137.)

As in this example $b$ is zero, the expression (B) is

$$
4 x^{2}+3 x-23
$$

and as the only doubtful interval, as ascertained by the partial analysis of Budan (ibid. p. 138), is the interval $[1,10]$, it is in reference to this alone that we have to examine the signs of the quadratic expression just written. Of this interval, the portion $[1,2]$, being negative, we reject it: taking therefore the interval $[3,10]$, which is wholly positive, and employing it, instead of the wider interval $[1,10]$, in Budan's analysis, a root is detected; and consequently, since no root can lie in the rejected region, the other root must lie in the interval $[2,3]$. And thus the character and places of the roots are ascertained.

It is proper to add, that if the second term be absent from the proposed equation (A), then the rational part of each conjugate factor will be

$$
x^{n}+\frac{1}{2} b x^{n-2}
$$

and the expression under the radical will be

$$
-c x^{2 n-3}+\left(\frac{1}{4} b^{2}-d\right) x^{2 n-4}-\ldots-k,
$$

to which all the preceding remarks apply.

I need scarcely observe, after what has been shown in my paper in the April Number of this Journal, that the conjugate factors here employed form but one pair, out of several that might be used for a similar purpose; although the forms here given will, as far as they go, be those most eligible; and I venture to think that, by our availing ourselves of them in 
the analysis of equations, considerable facilities will often be introduced into the numerical process. I shall only observe, in conclusion, that, by using other conjugate forms, it is easy to see how equations of an odd degree may be brought, in a direct manner, under the operation of the above method; or, without using any change of form at all, the method becomes immediately applicable upon introducing, into an equation of an odd degree, the new root $x=0$; or by supposing this to be done after the partial analysis of the equation in its original state.

But the general principle here sketched, admits of important modification. I shall hereafter show that, for an equation of the degree $2 n$, the polynomial under the radical may always be reduced to the degree $n-1$; and that for an equation of the degree $2 n+1$, it may be reduced to the degree $n+1$ : so that the analysis of a biquadratic equation will depend on the examination of an expression of the first degree; that of an equation of the fifth or sixth degree, upon the examination of an expression of the second degree; and so on.

In what is stated above, I have alluded to certain circumstances of doubt, of which no mention is made in the general announcement in this last paragraph. I have done so because, in this brief sketch of the initial steps of the investigation in which I am engaged, no explicit guidance in these circumstances is actually furnished. My present purpose is simply to make known the path I am pursuing in an important inquiry; and to point to the results which I confidently expect to arrive at.

Belfast, May 12, 1849.

LXII. On the Multiple Sounds of Bodies. By M. Duhamel, Member of the Institute*.

THE question under consideration is far from being new, 1 and still it may be said to be as yet unsolved; that is to say, scientific men have not yet arrived at a common and uncontested opinion on this point. The object of the present memoir is to establish such an opinion.

What follows will perhaps appear so evident, and so little different from what is already known, that persons who have for a long time been too easily satisfied on this subject, may, whilst they accept my explanation, remain persuaded that they have never viewed the matter otherwise. I expect this, and consent, if they will have it so, that all my ideas and ex-

* From the Annales de Chimie et de Physique for January 1849. 